\title{
Review of: "A Comprehensive Study On Soundness, Microstructure and Uniformity of 2024 Aluminum Cups Hydro-Mechanically Drawn at Elevated Temperatures"
}

\author{
yiwei zhang ${ }^{1}$ \\ 1 Anhui University of Technology
}

Potential competing interests: The author(s) declared that no potential competing interests exist.

The paper "A Comprehensive Study On Soundness, Microstructure and Uniformity of 2024 Aluminum Cups Hydro-Mechanically Drawn at Elevated Temperatures" is well written. It contents interesting experimental results and may be published after revision. Following comments may be recommended for revision version of the paper:

I don't feel qualified to judge about the English language and style, but I have spotted some problems and give you some suggestions to make it better. That is why your paper requires extensive editing of English language and style by qualified person.

After reading 4. Results and Discussion section I have got an impression that it contains sentences which sounds like in 'a revision' type of paper instead of 'a regular paper'. Decision making problem concerning the structure of the paper. Results and Discussion and then 5 Conclusions applied in the paper. I do recommend "Results, Discussion and then Conclusions." My advice is to rewrite sections 4 results and 5 discussions separately taking into account spotted lacks.

Page 4: "3. Finite-element Simulations": Why the authors take the punch, blank holder and the matrix as analytical rigid surfaces? Actually, the punch, blank holder and the matrix were the elastic deformation body. I recommend that give the reasons of the punch, blank holder and the matrix as rigid body in the manuscript.

What does the $\mathrm{H}(\mathrm{mm})$ in Figure 5 and Table 1 stand for? It should explain in the manuscript.

Page 10 on the first line: Why the authors take the micrograph of region A with maximum average grain size and region $C$ with minimum average grain size in Fig. 12? I think the picture cannot represent the average grain size for these two areas.

Page 11: The presence of copper precipitations in the material is likely to influencing the hardness. But, the 
energy dispersive spectroscopy (EDS) test cannot determine that the precipitations were $\mathrm{Al}_{2} \mathrm{Cu}$ in Fig.14. I think the precipitations should be calibrated by TEM diffraction spots in the manuscript.

I also do recommend you to download and study the following paper to make it better: Franco A. Maiorana; Horacio F. Mayer: "How to avoid common errors in writing scientific manuscripts". European Journal of Plastic Surgery (2018) 41:489-494. https://doi.org/10.1007/s00238-018-1418-z 